Faculty of Science

Faculty Publications

This is a post-review version of the following article:

Dynamic electrochemical impedance spectroscopy for electrocatalytic reactions

Robert L. Sacci, Frode Seland, David A. Harrington

June 2014

The final published version of this article can be found at:

https://doi.org/10.1016/j.electacta.2014.02.120

Citation for this paper:

Sacci, R.L., Seland, F. \& Harrington, D.A. (2014). Dynamic electrochemical impedance spectroscopy, for electrocatalytic reactions. Electrochimica Acta, 131, 13-19. https://doi.org/10.1016/j.electacta.2014.02.120 
(C) 2014. This manuscript version is made available under the CC-BY-NC-ND 4.0 license http://creativecommons.org/licences/by-nc-nd/4.0/

\title{
Dynamic electrochemical impedance spectroscopy for electrocatalytic reactions
}

\author{
Robert L. Sacci ${ }^{\mathrm{a}, 1}$, Frode Seland ${ }^{\mathrm{b}}$, David A. Harrington ${ }^{*, a}$ \\ ${ }^{a}$ Department of Chemistry, University of Victoria, Victoria, British Columbia, V8W 3V6, \\ Canada. \\ ${ }^{b}$ Department of Materials Science and Engineering, Norwegian University of Science and \\ Technology, NO-7491 Trondheim, Norway.
}

\begin{abstract}
Dynamic electrochemical impedance spectroscopy with the ac signal applied while sweeping the potential is a powerful method to study reaction mechanisms of surface electrochemical reactions. The ability to reliably measure low frequencies while sweeping is key to its successful implementation. Numerical modeling of a simple adsorption reaction is used to decide how fast the potential sweep can be at a given frequency. Careful baseline subtraction is crucial to achieve accurate results. Hardware and software implementing this method are described. With attention to issues of resolution and using the baseline correction algorithm described, accurate results can be obtained down to about 1 $\mathrm{Hz}$ under practical conditions. Sample experimental data is validated using the Kramers-Kronig transform and a consistency test involving the voltammogram slope and the low frequency data.
\end{abstract}

Key words: dynamic, EIS, impedance, FFT

\section{Introduction}

Dynamic electrochemical impedance spectroscopy (dEIS) has been implemented in various ways and used to study many different types of electrochemical systems, as reviewed recently [1]. Here the emphasis will be on using dEIS to study electrocatalytic and other surface electrochemical reactions, superimposing the ac on slow-sweep potentials. The question of how fast one can sweep at a given frequency and still measure valid EIS has been little discussed, and then mainly in terms of reversible solution electrochemistry. We investigate it here by numerical modeling of a simple adsorption reaction without any a priori

\footnotetext{
${ }^{*}$ Corresponding author. Tel.: +1-250-7217166

Email addresses: rsacci@uvic.ca (Robert L. Sacci), frode.seland@ntnu.no (Frode Seland), dharr@uvic.ca (David A. Harrington)

${ }^{1}$ Present address: Materials Science and Technology Division, Oak Ridge National Laboratory, Oak Ridge, TN 37831, USA.
}

Preprint submitted to Electrochim. Acta 
assumptions about the separability of the ac and dc signals. (Throughout this paper, dc will mean the slow sweep voltammetry condition; the term steady state will be reserved for the true dc condition.) Combined with some experimental validations, we show the method can be useful to frequencies as low as about $1 \mathrm{~Hz}$ under practical conditions, and is well suited to mechanistic analysis of electrocatalytic reactions.

The methods and conclusions here may be useful for studying other than electrocatalytic reactions, but use of dEIS to study surface reaction mechanisms has some special requirements that are rather stringent. To deduce the reaction mechanism, it should be investigated over a wide potential range (typically voltammograms range over a volt or more), and over a wide enough frequency range to measure all the time constants. Analysis in the complex plane (the "EIS" in dEIS) is virtually mandatory in such studies, which involve complex non-linear least-squares fitting of complex plane data to equivalent circuits or to impedance expressions of proposed reaction mechanisms. Early work evolved out of single-frequency ac voltammetry and was focused on analytical applications or measurement of double-layer capacitance, in which the objective was to find an analyte concentration or the pzc. In both cases the form of the frequency dispersion was known or assumed and single frequencies or simple plots vs square root of frequency were sufficient to analyze data; careful examination of the complex plane behavior was not required.

Smith and coworkers pioneered fast FFT methods [2-9], and others such as Bond [10, 11], Osaka [12], Popkirov [13-16], Rosvall [17, 18], Darowicki [19-23], Roy [24-29], Ragoisha [30-33], Hubin [34-38], Itagaki [39] and their coworkers have made advances through newer generations of computer technology and introduction of new algorithms. Smith's early work focused on fast measurements but not the dynamic aspect: the potential was stepped and then the measurement made after a settling time. Only later was voltammetry incorporated [7], and in only one case was the influence of sweep rate on the measurement considered [9]. Most subsequent workers preferred small potential steps, and continuous sweep FFT methods were popularized later by Darowicki [19-23], Roy [24-29] and Bond [11]. Ragoisha's potentiodynamic method introduced wavelets [30-33], but with $2 \mathrm{mV}$ steps at $2 \mathrm{mV} \mathrm{s}^{-1}$ is strictly a staircase waveform. Although small steps may approximate an analog sweep, the current spikes and then relaxes before measurement. The unmeasured current spike represents an unknown coverage change, which is detrimental in studying surface reactions.

New algorithms have been used to optimize the experimental impedance, such as Darowicki's short time fourier transform method [19] or wavelets [30]. The ability of these methods to analyze data in the complex impedance plane while sweeping is not different from the more prosaic method of running multiple ac voltammograms at different frequencies and assembling the data in complex plane, as we have been doing since 1993 [40-44], and as Shiu et al also did [45]. We have since updated to the FFT method described herein, but the key issue of how fast valid measurements can be made is common to all the sweep measurement methods. In common with most other workers we avoid the regime where the system is changing so fast with time that new concepts 
such as the rotating Fourier transform [46] or the Hilbert transform [47] are required, because then the supporting mechanistic theory is not available.

\section{Methods}

\subsection{Hardware}

The basic requirements to implement dEIS are a digital to analog (D/A) converter to generate a waveform, a potentiostat to apply it to the cell, analog to digital (A/D) converters to digitize both current and potential, and software to perform the Fourier transforms to convert to impedance. Accurate synchronization between the $\mathrm{D} / \mathrm{A}$ and $\mathrm{A} / \mathrm{D}$ channels is critical, and also the ability to continuously generate and acquire high-frequency data over the ten minutes or so required for a slow sweep voltammogram. The selected Keithley KUSB-3116 data acquisition system satisfies these requirements, with 16 bits per channel and a maximum sampling rate of $500 \mathrm{ksamples} / \mathrm{s}$. The A/D channels are sampled successively and not simultaneously, which potentially gives rise to a phase error. However, for ac signals of known frequency this may be corrected for exactly $[12,25,48]$. In order to avoid a staircase approximation to a sweep, an external analog sweep generator is used, and a Gamry potentiostat is operated as an analog potentiostat. The detailed implementation of the hardware has already be given [48]. We used a slight modification of the set of multisine frequencies proposed by Popkirov and Schindler [14], all multiples of a minimum (base) frequency at 13 frequencies per decade, typically 45 frequencies between ca $1 \mathrm{~Hz}-13 \mathrm{kHz}$. Amplitudes were chosen by Popkirov's 2:10 scheme [14], in which the amplitudes drop off exponentially by a factor or two for each decade in frequency. Phases were randomized but not optimized.

Loss of resolution in applying and acquiring the ac potential multisine was minimized by generating the multisine at $-10 \mathrm{~V}$ to $+10 \mathrm{~V}$, and then using an analog divider before adding to the analog sweep. The measured ac $+\mathrm{dc}$ potential ouput from the potentiostat first had the known dc sweep potential subtracted before amplifying back to full scale and digitizing. The system had the capability to generate the waveform at a faster clock speed than the A/D sampling to minimize quantization noise, but this was found to have little advantage. In practice the three channels (ac voltage, dc voltage and ac $+\mathrm{dc}$ current) were sampled continuously and synchronously with the generation of the multisine waveform. The custom software for control of the Keithley and processing of the data was written in Microsoft Visual Basic ${ }^{\circledR}$ v 6.

\subsection{Numerical solution of the differential equation}

Full numerical simulation of the response of the system to dc sweep + ac is used, without assumptions about the ac amplitude being small or the ac and dc being decoupled. This allows comparison with experiment in which discrepancies are due to the kinetic assumptions and not the inaccuracies of the theory. Such full simulations were first used for reversible solution reactions by Bond et 


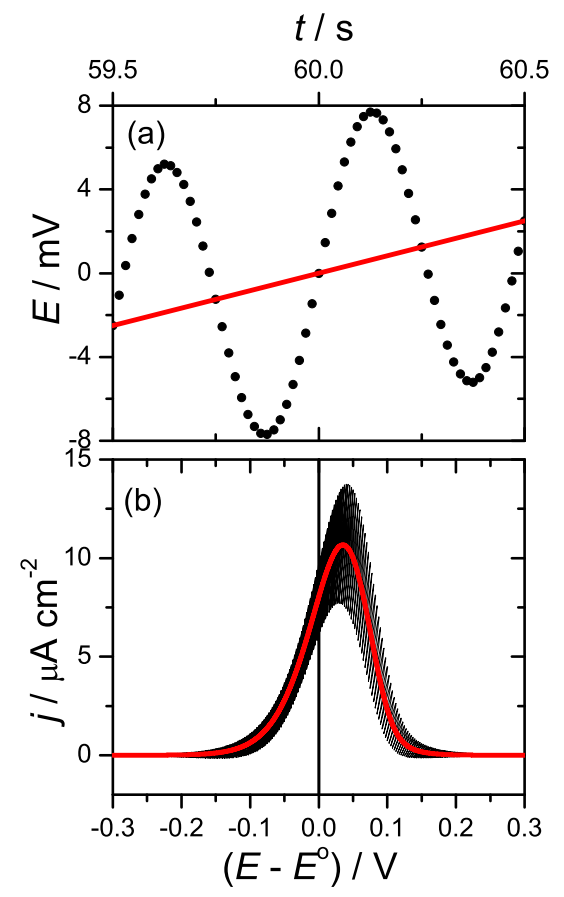

Figure 1: Waveforms for typical conditions. (a) $2 \mathrm{~Hz} 5 \mathrm{mV} \mathrm{rms}+5 \mathrm{mV} / \mathrm{s}$ applied potential, with each output point shown (circles, 64 points per second). Red line is the dc sweep only. (b) Simulated cyclic voltammograms for the standard parameters with ac (black) and without ac (red).

al [9] and Gavaghan and Bond [49], and more recently for mechanisms including surface reactions $[50,51]$. Here, the kinetic differential equation was solved using Maple ${ }^{\mathrm{TM}}$ for the ac $+\mathrm{dc}$ potential waveform of interest. The implicit Rosenbrock third-fourth order Runge-Kutta solver for stiff equations with degree three interpolant was used [52], with absolute and relative error tolerances of $10^{-6}$. For the $2 \mathrm{~Hz} 5 \mathrm{mV}$ rms single-frequency data reported here, 192 solver outputs per second were produced, but as the waveform was implemented as a function, the solver had access to times between these as required. Every third solved point of the current was output with every third point of the potential to mimic the sequential channel acquisition of the Keithley digitizer (Fig. 1(a)). This was then baseline corrected (see below) and Fourier transformed by the same software used for experimental data, with Fourier transforms of 32 points performed every $2.5 \mathrm{mV}$. (This data density is much lower than for real experiments, where much higher frequencies are also involved.) To calibrate the accuracy of the system, a steady-state system at $E^{\circ}$ (with the standard rate parameter set given below) gave an answer agreeing with theory to $0.2 \%$ for resistance and $0.02 \%$ for capacitance. This error may arise from the solver, the transform software, or the fact that the theory is strictly for small perturbation 
signals, but is more than sufficient to investigate the dynamic effects and is less than typical experimental errors. Preliminary calculations have been published [53].

\section{Results and discussion}

Our investigation of the importance of dynamic effects was done for the test case of a simple adsorption reaction (1), with Langmuir kinetics, Eqs. (2) - (6).

$$
\begin{aligned}
& \underset{1-\theta}{\mathrm{M}(\text { site })}+\mathrm{A}^{-}(\mathrm{aq}) \rightleftarrows \underset{\theta}{\mathrm{MA}(\mathrm{ads})}+\mathrm{e}^{-} \\
& \Gamma_{\mathrm{m}} \frac{d \theta}{d t}=v_{1}=k_{1}(1-\theta)-k_{-1} \theta \\
& k_{1}=k^{o} \exp \left(\frac{\left(1-\beta_{1}\right) F\left(E-E^{o}\right)}{R T}\right) \\
& k_{-1}=k^{o} \exp \left(-\frac{\beta_{1} F\left(E-E^{o}\right)}{R T}\right) \\
& E=E_{\mathrm{i}}+v t+a \sin (\omega t) \\
& j_{\mathrm{f}}=F v_{1}
\end{aligned}
$$

Here, $\Gamma_{\mathrm{m}}$ is the concentration of sites on the clean surface in $\mathrm{mol} \mathrm{m}^{-2}$. Fast mass transport and unit concentration of $\mathrm{A}^{-}$is assumed. Unless specified otherwise, the standard parameters and conditions were: $5 \mathrm{mV} \mathrm{s}^{-1}$ sweep rate, $2 \mathrm{~Hz} 5$ $\mathrm{mV}$ rms ac signal, $\Gamma_{\mathrm{m}}=2.5 \mathrm{nmol} \mathrm{cm}{ }^{-2}, k^{o}=2 \times 10^{-10} \mathrm{~mol} \mathrm{~cm}^{-2} \mathrm{~s}^{-1}, \beta_{1}=$ $0.5, T=298.15 \mathrm{~K}$. This reaction has a steady state current of zero at any given potential, and the linear sweep voltammetry peak (Fig. 1(b)) is a purely transient current, eminently suitable for testing dynamic effects. The theoretical faradaic impedance is a series combination of a charge-transfer resistance and pseudocapacitance, given by Eqs. (7) and (8) [54].

$$
\begin{aligned}
R_{\mathrm{ct}}^{-1} & =F\left(\partial v_{1} / \partial E\right)_{\theta} \\
C & =-\Gamma_{\mathrm{m}} F\left(\partial v_{1} / \partial E\right)_{\theta} /\left(\partial v_{1} / \partial \theta\right)_{E}
\end{aligned}
$$

The differential equation (2) was solved for the chosen parameters, and the faradaic current, Eq. (6), and potential were output to the calculation program, which calculated the faradaic impedance via Fourier transformation in the usual way. This "experimental" impedance $Z_{\text {expt }}$ yielded an experimental $R_{\text {ct }}=\operatorname{Re}\left(Z_{\text {expt }}\right)$ and $C=-1 / \omega \operatorname{Im}\left(Z_{\text {expt }}\right)$. These were compared with the theoretical values above, where the partial derivatives were calculated from the coverages calculated by solving the differential equation for the same sweep conditions without the ac signal. Note that the coverages determined in this way are different from the coverages that would be found in a steady state experiment at the same potential. Although Eqs. (7) and (8) use the correct surface coverage, they are not otherwise "aware" that this is a dynamic experiment, because of the small perturbation assumption. 


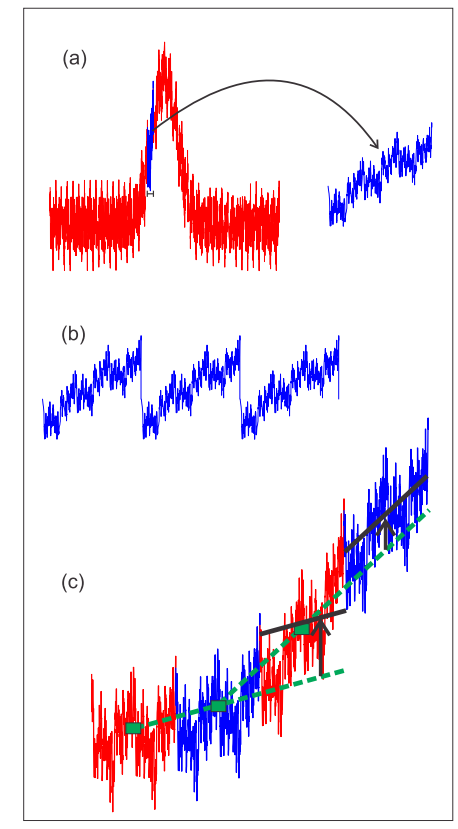

Figure 2: Baseline problem and solution. (a) a section of current (blue) has a significant slope, which the Fourier transform sees as a periodically repeated "sawtooth" waveform (b). (c) Extrapolation baseline estimates the slope (dashed line) from the dc values (green squares) of the two previous sections. The height of the baseline is adjusted (arrows) to give the black baseline, which does not alter the dc current. Note that the sections here are much exaggerated in length and slope compared to the real situation.

\subsection{Baseline corrections}

In real experiments, we noticed that large errors occurred unless baseline corrections were made before Fourier transformation of the current. For the potential, the dc sweep was subtracted electronically before digitization, but the current without the ac signal is not known and so cannot be subtracted in this way. The current could be treated with a low pass filter, but any remaining higher frequencies would lead to errors in the ac accuracy, so we instead implemented a software strategy. Fig. 2 shows why baseline correction is required. The segment of the current waveform to be transformed (one period of the base frequency, blue in (a)) is treated by the Fourier transform as periodically extended as shown in (b). Such a sawtooth waveform has significant contributions to the fundamental (base) frequency and its harmonics, and so it is necessary to subtract a sloping baseline. Schemes of considerable complexity are possible, but one constraint is that the software should be able to make only one pass through the data for efficiency reasons. It is also desirable that the baseline uses many data points rather than just a few, in order to minimize susceptibility to noise. Since the Fourier transform itself uses many data points to accurately find the dc value of a waveform, our strategy, which we refer to as "extrapolate", is to estimate the slope from the dc values of the two previously-transformed 


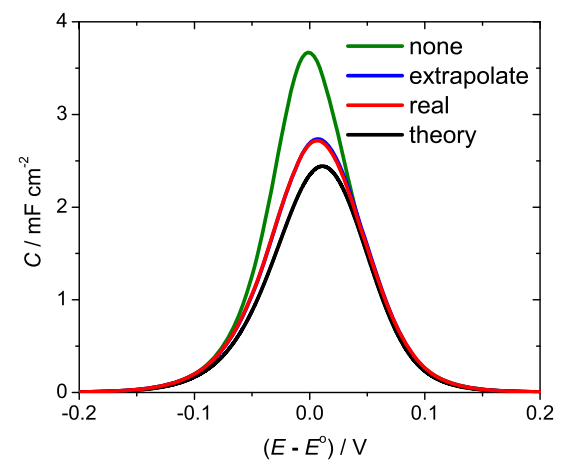

Figure 3: Baseline effects on the capacitance. The "extrapolate" baseline correction (blue) is largely hidden behind the "real" baseline correction (red). Both of these give results significantly different from the theory at this sweep rate, which proves to be too fast.

sections, as in Fig. 2(c). A line of this slope is adjusted in height so that it does not affect the dc value, and is used as the baseline. Fig. 3 shows that the extrapolate correction is a dramatic improvement over no baseline correction.

Evidently, baseline correction is crucial to getting accurate results. As Bond noted in his simulation of the reversible solution case [9], subtraction of the real dc current before transformation greatly increases the accuracy, and he suggested running a separate cycle without ac to use as a baseline. This is not optimum as an experimental strategy because the system must be accurately reproducible over long times. However, in the case of the simulation it is easy to subtract the current found from the differential equation without applied ac, in order to know what the "real" baseline is. The "extrapolate" baseline corrected and "real" signals are nearly overlaid on the figure, showing that the extrapolate baseline is an effective solution. There is still a small discrepancy, which for these rate parameters maximized at about $1.5 \%$ just past the peak. However, the real and baseline-corrected curves both differ significantly from the theoretical value of the capacitance (black curve), being about $10 \%$ different at the peak. This error is the result of sweeping too fast, as we now demonstrate.

\subsection{Errors due to sweep rate}

We first note that tests of the dEIS system on dummy cells showed that correct values of the parameters were obtained up to at least $50 \mathrm{mV} \mathrm{s}^{-1}$ [48]. Evidently the errors seen here are due to the inherently non-linear nature of electrochemical systems, and are not adequately probed by dummy cells, for which the steady-state current-potential relation is linear. The effect of sweep rate was investigated by sweeping five times faster or slower than above, using the same rate constant (Fig. 4). At $1 \mathrm{mV} \mathrm{s}^{-1}$, the capacitance error is less than $1 \%$, though the resistance still shows some error. In contrast, at $25 \mathrm{mV} \mathrm{s}^{-1}$ it is not possible to make a sensible measurement of capacitance, error prone or not. The period of a cycle now covers a large change in dc current. It is interesting 


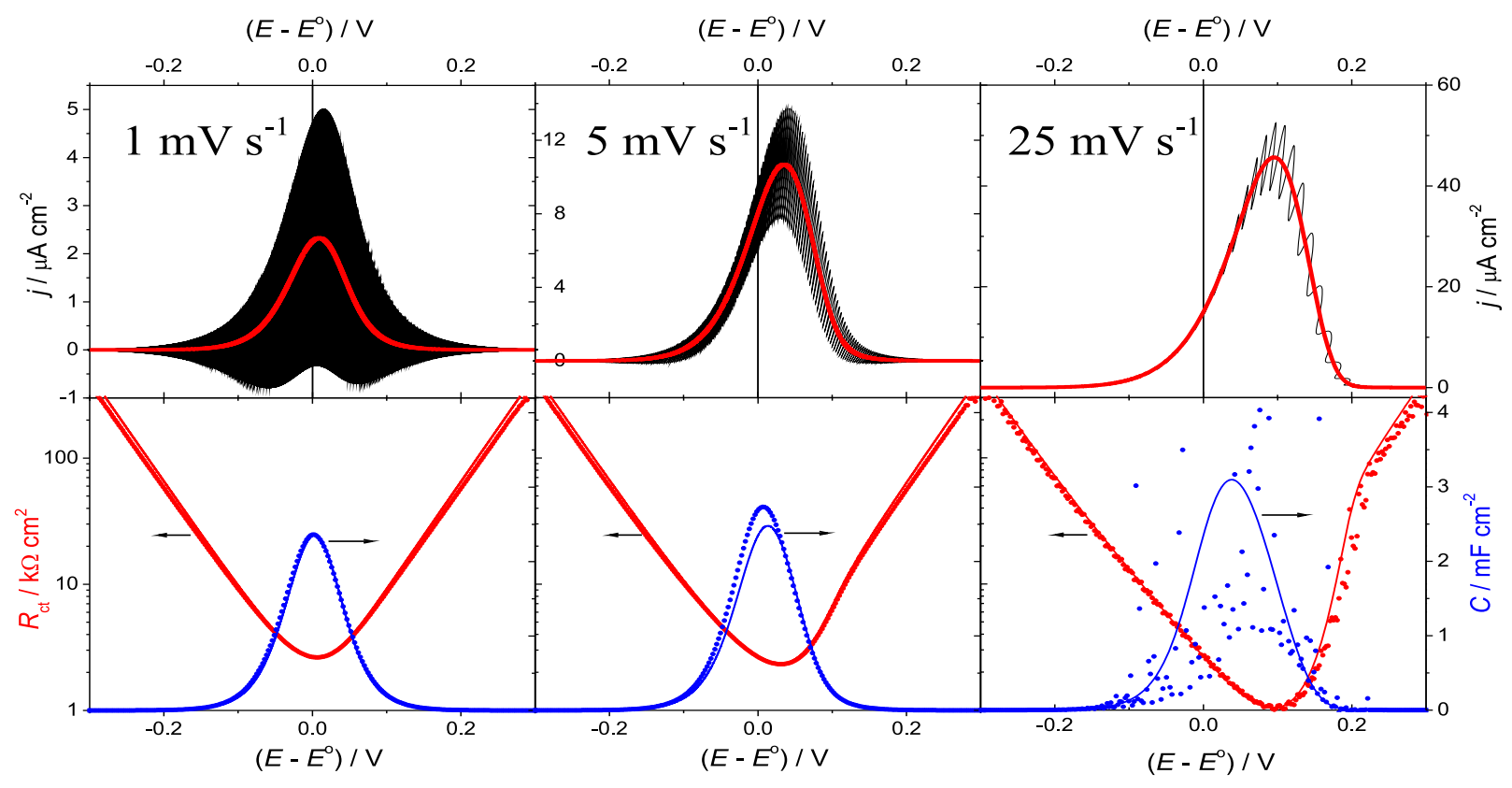

Figure 4: Sweep rate effect. Top: simulated cyclic voltammograms for the standard parameters and quoted sweep rates with ac (black) and without ac (red). Bottom: $R_{\mathrm{ct}}$ and $C$ after determined with the extrapolate baseline (circles) compared with theory (lines).

to note that the accurate results at $1 \mathrm{mV} \mathrm{s}^{-1}$ are achieved despite the fact that the ac current amplitude (black envelope) is comparable to the dc current.

There is a general expectation that the sweep potential should not change much during an ac cycle, i.e., the slow-scan limit should apply. The change at $5 \mathrm{mV} \mathrm{s}^{-1}$ depicted in Fig. 1(a) is $35 \%$ of the (peak) amplitude and intuitively seems to be too much, but at $1 \mathrm{mV} \mathrm{s}^{-1}$, the change is less than $10 \%$. This is in accord with the observed errors just discussed. Underkofler and Shain [55] were the first to discuss the validity of the slow-scan limit, concluding from the analytical equation for a reversible solution reaction that the angular frequency and sweep rate should be related by

$$
\omega \gg v / \Delta E
$$

where $\Delta E$ is the amplitude. If "much greater" means a factor of ten, then this is satisfied for both the $1 \mathrm{mV} \mathrm{s}^{-1}$ and $5 \mathrm{mV} \mathrm{s}^{-1}$ sweeps above $(\omega=12.6 \mathrm{rad}$ $\mathrm{s}^{-1}$ at $2 \mathrm{~Hz}$ ). We suggested [40], based on an analytical expression for a surface reaction, that

$$
\omega \gg v(F / R T)
$$

which would allow lower frequencies. Both these estimates come from requiring one term in an analytical expression to be much less than the next, without any numerical estimates. Bond et al [9] were the first to make numerical estimates 
(for the reversible solution case) and estimated that a dc change of $3.2 \mathrm{mV}$ when the ac amplitude was $3.5 \mathrm{mV}$ rms was acceptable for the $15.6 \mathrm{~Hz}, 50 \mathrm{mV}$ $\mathrm{s}^{-1}$ conditions simulated. This seems large, but is consistent with Eq. (9). Henderson and Gordon [56] used an analytical solution but without a priori ac and dc separation and found that $\omega \gtrsim 10 n F v / R T$ with a $5 \%$ error. Both works agreed that significant changes in dc during an ac cycle could be tolerated. Later treatments of the reversible case were more focused on large amplitude effects $[49,57,58]$. Although these reversible solution cases are likely not fully relevant to the surface case, the idea that reasonable results may be obtained with significant changes in dc during an ac cycle is probably generally true. Garland et al [25] later discussed the experimental constraints of FFT-dEIS and used Eq. (9) with the amplitude replaced by the rms amplitude. They pointed out that there should be an equivalent constraint in the current,

$$
\omega \gg\left(d j_{\mathrm{dc}} / d t\right) / \Delta j_{\mathrm{rms}}
$$

where $\Delta j_{\mathrm{rms}}$ is the rms amplitude of the ac current density response. They made the important point that this is system dependent and so is not known in advance of the experiment. In the present case, at $5 \mathrm{mV} \mathrm{s}^{-1}$ the right-hand side of Eq. (11) takes the value 0.72 at the potential on the trailing edge of the peak where the slope $\left(d j_{\mathrm{dc}} / d t\right)$ is maximum, and this constraint is satisfied. However, $\left(d j_{\mathrm{dc}} / d t\right)$ increases with sweep rate but $\Delta j_{\mathrm{rms}}$ is approximately constant, and for $25 \mathrm{mV} \mathrm{s}^{-1} \omega$ is only twice the right-hand side, which suggests that this may be a limiting condition. We propose that other system variables such as concentration or coverages must be likewise constrained. In the present case, the coverage should be constrained by

$$
\omega \gg(d \theta / d t) / \Delta \theta_{\mathrm{rms}}
$$

This is not only system dependent, but is also not available from a typical electrochemical experiment. At $5 \mathrm{mV} \mathrm{s}^{-1}$, the right-hand side is 64 at the peak, so this is apparently the most severe constraint. However, at $1 \mathrm{mV} \mathrm{s}^{-1}$ the right-hand side is 15 and the constraint is still violated even though good results are obtained. Evidently, a simple condition for valid results remains to be found. The system-dependent current and coverage constraints are governed by the rate constant and variation of this is discussed below.

\subsection{Transform length effects}

Most authors assume that the periods transformed (here 1 cycle of $2 \mathrm{~Hz}$ $=0.5 \mathrm{~s}$ ) follow each other, so that the interval between successive transforms is the same as the transform period, as suggested by Fig. 2. However, the interval can be chosen to be longer or shorter than the transform period; both choices are used in Fig. 4. Shorter intervals give overlapping transforms, with correspondingly higher data density, though the resolution is expected to depend on the transform period. In cases where the period of the base frequency is

much shorter than the data density interval required, several periods may be 


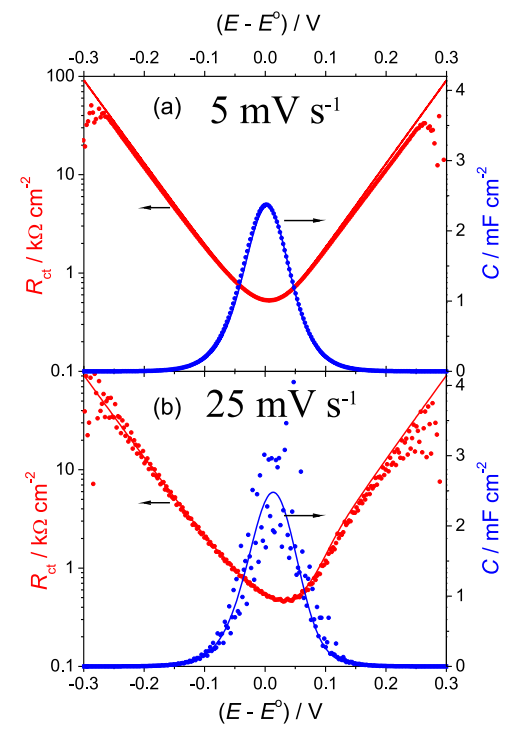

Figure 5: Rate constant effect. Five times larger rate constant than in Fig. $4, k^{o}=1 \times 10^{-9}$ $\mathrm{mol} \mathrm{cm}-2 \mathrm{~s}^{-1}$.

transformed together. This degrades the resolution, but improves the signal-tonoise ratio.

The implicit assumption in the above discussion of sweep rate effects is that slowing the sweep rate to $1 \mathrm{mV} \mathrm{s}^{-1}$ led to acceptable results because the system variables changed less during an ac cycle. However, reducing the sweep rate reduced the transform period from $2.5 \mathrm{mV}$ wide at $5 \mathrm{mV} \mathrm{s}^{-1}$ to $0.5 \mathrm{mV}$ wide, which means that the system variables changed less in any case. Transforming 8 periods together at $1 \mathrm{mV} \mathrm{s}^{-1}$ makes the transform $4 \mathrm{mV}$ wide, wider than at $5 \mathrm{mV} \mathrm{s}^{-1}$, but with almost no change in the capacitance, so the capacitance error at $5 \mathrm{mV} \mathrm{s}^{-1}$ is a true sweep rate effect and not a transform length effect. Transforming 16 periods at $1 \mathrm{mV} \mathrm{s}^{-1}$ changes the capacitance by only $1 \%$, and only by going from 16 periods to 32 periods is the peak capacitance reduced below the theoretical value, indicating significant oversmoothing of the peak.

\subsection{Rate constant effects}

The effect of increasing the rate constant by a factor of five is shown in Fig. 5. Comparing the $5 \mathrm{mV} \mathrm{s}^{-1}$ results with those in Fig. 4, it is evident that the error in the capacitance has been reduced, and that it is now comparable to that at $1 \mathrm{mV} \mathrm{s}^{-1}$ for the lower rate constant. Sensible results are now found at $25 \mathrm{mV}$ $\mathrm{s}^{-1}$, and the results resemble those at $5 \mathrm{mV}^{-1}$ at the lower rate constant, albeit with increased noise. That five times the rate constant gives the same behaviour at five times the sweep rate is strictly true for the dc behavior of this system, since dimensional analysis shows that the dc behavior depends on $v / k^{o}$, and this appears to also govern the deviation of the measured capacitance from the 
theoretical value. This is consistent with the discussion above suggesting that the change in dc voltage during an cycle can be violated without appreciable error in some cases; it is five times worse here and the results are noisier but the deviation is less.

The strong dependence on rate constant invalidates simple system independent criteria like Eqs. (9) and (10). The current constraint, Eq. (11), can be determined from the experimental results but not predicted beforehand, but it appears to lack predictive value. Another parameter that can be determined from the experimental results without knowing the surface coverage is the smallest time constant. Here, the time constant $\tau=R_{\mathrm{ct}} C$ is maximum (for $\beta_{1}=1 / 2$ ) at $E=E^{o}$, where its value is $\Gamma_{\mathrm{m}} / 2 k^{o}$. For $k^{o}=2 \times 10^{-10} \mathrm{~mol} \mathrm{~cm}^{-2} \mathrm{~s}^{-1}$, $\tau=6.25 \mathrm{~s}$ and for $k^{o}=1 \times 10^{-9} \mathrm{~mol} \mathrm{~cm}{ }^{-2} \mathrm{~s}^{-1}, \tau=1.25 \mathrm{~s}$. Since $\tau$ is an inverse measure of the rate constant, and the error depends on $v / k^{o}$, the error should depend on $\tau v$. If $\tau$ is the time constant for the slowest process (the largest $\tau$ ), we speculate that frequencies $\omega$ down to about $\tau^{-1}$ can be measured provided that

$$
\tau v(F / R T) \lesssim 1
$$

The quantity $\tau v(F / R T)$ is 0.2 and 1 for Figs. 5a and b respectively. Although this condition appears to work here, it should be further tested on other cases.

\subsection{Validation in practice}

The simulations above are useful for determining which effects are significant in a dEIS experiment, but validation of the method for real data is worth presenting here. We first point out that for most of the electrocatalytic systems that we and others have investigated, data fitted well to equivalent circuits that are expected for mechanisms involving adsorbed intermediates. In cases where the circuit elements are positive capacitors, resistors and inductors, the Kramers-Kronig (KK) relationships will apply. Such linear, lumped circuits are known to satisfy the "positive real" (PR) condition [59], which is derived from the requirement that the energy stored in capacitors and inductors must be positive, and that power is dissipated in resistors. The PR condition implies stability [59], and such circuits are causal since they are governed by differential equations in time. The power and energy constraints mean that their outputs must be finite, and so they satisfy all the conditions for KK to hold (linear, causal, stable and finite). See [60] p. 400 for details showing that PR implies KK. The literature on circuits with negative elements is more confusing however. Gabrielli showed that to satisfy the stability criterion, one must apply the KK transform in the transfer function plane appropriate to the type of control, i.e., the admittance plane for potentiostatic control [61], and this was more recently emphasized by Sadkowski et al $[62,63]$. Our strategy has been to fit dEIS data to circuits for which the poles of the admittance are stable. Some skepticism of this procedure remains, especially when impedances with negative real parts are present, and so we present here a typical KK test using Boukamp's method $[64,65]$ for formic acid oxidation dEIS data on Pd [66] reaching into the second quadrant (Fig. 6). Similar KK validation of ethanol oxidation dEIS data was 


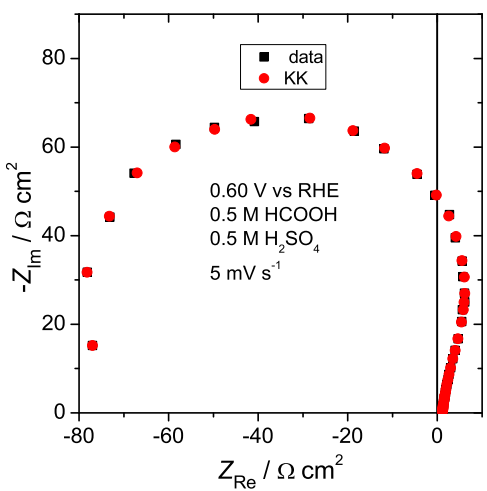

Figure 6: Kramers-Kronig test for formic acid oxidation data. Test in the admittance plane using Boukamp's method with 7.05 time constants per decade. Real and imaginary residuals are randomly distributed (not shown). Lowest frequency shown is $1 \mathrm{~Hz}$.

shown recently [67]. Evidently, perfectly acceptable results are available at 1 $\mathrm{Hz}$ and $5 \mathrm{mV} \mathrm{s}^{-1}$ under practical conditions.

Although steady states as measured in conventional EIS must be stable (or steady state would not be achieved), cyclic voltammetry captures transient states that can be unstable. For example, voltammetry has been widely used to study nucleation and growth of films, even though it is unstable in the growth phase. Dynamic EIS can measure such processes, as we have found for methanol oxidation [43], and parameters deduced from dEIS may be expected to be approximately correct, as we have shown in simulations [53].

A limiting aspect of dEIS is that frequencies lower than about $1 \mathrm{~Hz}$ are not easily measured. Aside from questions of validity related to sweep rate, our experience is that noise dominates lower frequency measurements. It becomes an important question then about whether slower processes exist but have not been observed. The relationship between the zero-frequency intercept of the impedance and the slope of the polarization curve, Eq. (14) has been used as a consistency check of impedance data for a long time [68]; a proof and further discussion is given in Ref. [69].

$$
\lim _{\omega \rightarrow 0} Z(\omega)=\left(\frac{d j_{\mathrm{dc}}}{d E}\right)^{-1}
$$

Here we assume that the appropriate "dc" current-potential relationship is the cyclic voltammogram; processes too slow to show up in voltammetry will not show up in dEIS either. Fig. 7 shows an approximate test of this relationship, where data at a low frequency is taken as an approximation of the limit, and the voltammetry current is numerically differentiated to give the right-hand side. Good agreement is obtained for most of the voltammogram. In particular, the region at potentials less than about $0.7 \mathrm{~V}$ where formic acid is oxidized on an oxide-free surface can be reliably studied by dEIS. As in other systems we have studied, there is some discrepancy in the oxide growth and reduction regions at 


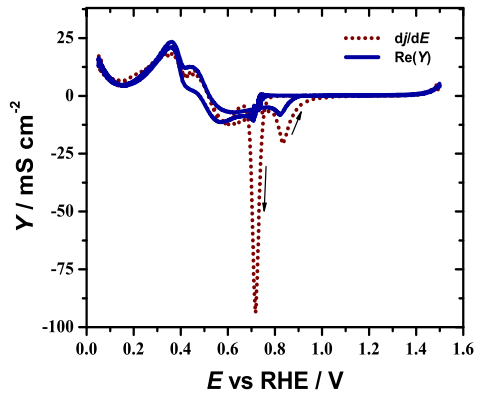

Figure 7: Low frequency validation test. Data for oxidation of formic acid on Pd. $0.5 \mathrm{M}$ $\mathrm{HCOOH}, 0.5 \mathrm{M} \mathrm{H}_{2} \mathrm{SO}_{4}$. Dotted curve: numerical differentiation of $10 \mathrm{mV} \mathrm{s}^{-1}$ voltammogram. Solid curve: Real part of admittance at $5 \mathrm{~Hz}$.

higher potentials; it appears that sudden removal of the oxide on the reverse sweep at about $0.7 \mathrm{~V}$ leads to competition for freely vacated sites that is not properly captured by dEIS. Note that our test reaction simulations do not obey this condition (Fig. 4), since the voltammogram has a negative slope but the impedance is never negative. This is a case where the true steady-state currentpotential relationship has zero slope (zero current at all potentials), and satisfies Eq. (14) (the impedance is infinite at zero frequency), but the transient current has a qualitatively different shape. The oxide growth also has a true steady-state current that is zero (or very small), which may explain the poor agreement in that region also. These cases where the steady-state current is zero are atypical and we may expect agreement in the general case, though further investigation of the theoretical basis of this equation for sweep conditions is required.

\section{Prospects}

The key advantage of dEIS is to apply the the power of complex-plane analysis to surface conditions not accessible at steady state, which leads to better elucidation of surface reaction mechanisms. Additionally, the large density of data (spectra every few millivolts) collected rapidly leads to "big data" advantages for data analysis. This is illustrated with the set of " $R_{\mathrm{ct}}$ voltammograms" in Fig. 8. This shows the results of 2500 fitted spectra for a series of experiments in which the reversal potential was systematically changed. The forward sweep reaction is the formation of the platinum oxide film, which is reduced back to $\mathrm{Pt}$ on the reverse sweep in the peak around $0.8 \mathrm{~V}$. The feature extraction advantage is evident, e.g., the fine structure in the peak around 0.95 $\mathrm{V}$ is a reproducible feature that likely would be missed in a conventional experiment. In dc voltammetry, fine structure in this region has been attributed to resolved adsorption sites [70]. The common return sweep behavior (shown with the black arrow) indicates a surprising lack of dependence on coverage, an observation that can immediately simplify a more quantitative mechanistic 


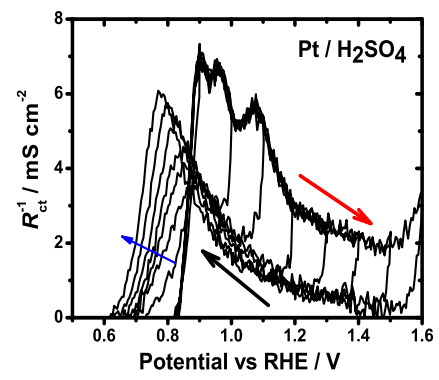

Figure 8: $R_{\mathrm{ct}}$ voltammograms with different reversal potentials. $R_{\mathrm{ct}}^{-1}$ from fitting to a circuit with $\left(R_{\mathrm{ct}}\right.$ in parallel to a CPE for the double-layer) in series with the solution resistance. Sweep rate $5 \mathrm{mV} \mathrm{s}^{-1}$. $0.5 \mathrm{M} \mathrm{H}_{2} \mathrm{SO}_{4}$. Red and black arrows indicate sweep direction; blue arrow indicates increasing reversal potential.

analysis (forthcoming). The most interesting potential advantage of the high data density is to enable model-free deduction of rate laws. For example, if Nyquist plots show the two-semicircle shape that indicates a surface mechanism with a single adsorbed species, we may postulate a very generic rate law such as the product of some coverage dependence, some potential dependence and some concentration dependence for the rate of production of electrons and the rate of production of the adsorbed species:

$$
\begin{aligned}
j / F=r_{\mathrm{e}} & =g_{\mathrm{e}}(\theta) f_{\mathrm{e}}(E) h_{\mathrm{e}}(c) \\
r_{\theta} & =g_{\theta}(\theta) f_{\theta}(E) h_{\theta}(c)
\end{aligned}
$$

Application of the definition of $R_{\mathrm{ct}}=\left(\partial r_{\mathrm{e}} / \partial E\right)_{\theta, c}^{-1}$ leads to

$$
\frac{j \cdot R_{\mathrm{ct}}}{F}=\frac{f_{\mathrm{e}}(E)}{f_{\mathrm{e}}^{\prime}(E)}
$$

which allows deduction of the potential dependence $f_{\mathrm{e}}(E)$ independently of the coverage or concentration dependence. Similar tests for the coverage or concentration dependence can lead to deduction of the rate laws (15) and (16). This avoids the trial and error process of testing candidate reaction mechanisms one at a time, which is frequently limiting in a conventional analysis.

\section{Conclusions}

The key issue in dynamic EIS is how low the frequency can be at a given sweep rate. Consideration of a model electrosorption reaction shows that proper baseline correction is crucial to achieve accurate results. The "extrapolate" baseline correction described here allows measurements down to frequencies similar to or lower than predicted by literature estimates. However, the lowest valid frequency at a given sweep rate is dependent on the rate constant(s) and is thus 
system dependent, making it hard to predict in the general case. Nonetheless, the system time constants can be deduced from the measured spectra without knowledge of the mechanism or rate constants, and are likely to be a better way to decide validity. Kramers-Kronig tests and tests of the low-frequency intercept against the slope of the voltammogram show that typical experimental dEIS data can be reliably used down to about $1 \mathrm{~Hz}$ at slow sweep rates (ca. 1-10 $\mathrm{mV} \mathrm{s}^{-1}$ ). In our experience, instrumental or other sources of noise limit use at lower frequencies.

The dynamic nature of the measurement allows more complete investigation of reaction mechanisms by accessing surface conditions that are not available at steady-state. The high density of data promises more reliable data analysis as well as the possibility of model-free analysis of reaction mechanisms.

\section{Acknowledgements}

We thank the Natural Sciences and Engineering Research Council of Canada, the Research Council of Norway, the University of Victoria, and the Norwegian University of Science and Technology (NTNU) for financial support. RLS thanks the University of Victoria for a Nora and Mark DeGroutier Memorial Scholarship.

\section{References}

[1] A.S. Bandarenka, Analyst 138 (2013) 5540.

[2] S.C. Creason, D.E. Smith, J. Electroanal. Chem. 40 (1972) A1.

[3] S.C. Creason, J.W. Hayes, D.E. Smith, J. Electroanal. Chem., 47 (1973) 9.

[4] D.E. Smith, Anal. Chem. 48 (1976) 221A.

[5] R.J. Schwall, A.M. Bond, D.E. Smith, Anal. Chem. 49 (1977) 1805.

[6] R.J. Schwall, A.M. Bond, D.E. Smith, J. Electroanal. Chem. 85 (1977) 217.

[7] A.M. Bond, R.J. Schwall, D.E. Smith, J. Electroanal. Chem. 85 (1977) 231.

[8] R.J. O'Halloran, J.C. Scharr, D.E. Smith, Anal. Chem., 50 (1978) 1073.

[9] A.M. Bond, R.J. O'Halloran, I. Ruzic, D.E. Smith, J. Electroanal. Chem., $90(1978) 381$.

[10] J. Hazi, D.M. Elton, W.A. Czerwinski, J. Schiewe, V.A. Vicente-Beckett, A.M. Bond, J. Electroanal. Chem. 437 (1997) 1.

[11] A.M. Bond, N.W. Duffy, S-X. Guo, J. Zhang, D. Elton, Anal. Chem. 77 (2005) 186A.

[12] T. Osaka, K. Naoi, Bull. Chem. Soc. Japan 55 (1982) 36. 
[13] G.S. Popkirov and R.N. Schindler, Rev. Sci. Instr. 63 (1992) 5366.

[14] G.S. Popkirov and R.N. Schindler, Rev. Sci. Instr. 64 (1993) 3111.

[15] G.S. Popkirov, E. Barsoukov, J. Electroanal. Chem. 383 (1995) 155.

[16] G.S. Popkirov, Electrochim. Acta 41 (1996) 1023.

[17] M. Rosvall, M. Sharp, Electrochem. Commun. 2 (2000) 338.

[18] M. Rosvall, Electrochem. Commun. 2 (2000) 791.

[19] K. Darowicki, P. Ślepski, J. Electroanal. Chem. 547 (2003) 1.

[20] K. Darowicki, J. Electroanal. Chem. 486 (2000) 101.

[21] K. Darowicki, J. Orlikowski, G. Lentka, J. Electroanal. Chem. 486 (2000) 106-110.

[22] K. Darowicki, S. Krakowiak, P. Ślepski, Electrochim. Acta 49 (2004) 2909.

[23] S. Krakowiak, K. Darowicki, P. Ślepski, J. Electroanal. Chem. 575 (2005) 33.

[24] M.J. Walters, J.E. Garland, C.M. Pettit, D.S. Zimmerman, D.R. Marr and D. Roy, J. Electroanal. Chem. 499 (2001) 48.

[25] J.E. Garland, C.M. Petit and D. Roy, Electrochim. Acta, 49 (2004) 2623.

[26] C.M. Pettit, J.E. Garland, M.J. Walters, D. Roy, Electrochimica Acta 49 (2004) 3293.

[27] V.R.K. Gorantla, K.A. Assiongbon, S.V. Babu, and D. Roy, J. Electrochem. Soc. 152 (2005) G404.

[28] S.B. Emery, J.L. Hubbley, D. Roy, Electrochim. Acta 50 (2005) 5659.

[29] C.M. Pettit, P.C. Goonetilleke, D. Roy, J. Electroanal. Chem. 589 (2006) 219.

[30] G.A. Ragoisha, A.S. Bondarenko, Electrochem. Comm. 5 (2003) 392.

[31] G.A. Ragoisha, A.S. Bondarenko, Surface Sci. 566-568 (2004) 315.

[32] G.A. Ragoisha, A.S. Bondarenko, Electrochim. Acta 50 (2005) 1553.

[33] G.A. Ragoisha, N. P. Osipovich, A. S. Bondarenko, J. Zhang, S. Kocha, A. Iiyama, J. Solid. State Electrochem. 14 (2010) 531.

[34] O.L. Blajiev, R. Pintelon, A. Hubin, J. Electroanal. Chem. 576 (2005) 65.

[35] O.L. Blajiev, T. Breugelmans, R. Pintelon, A. Hubin, Electrochim. Acta $51(2006) 1403$. 
[36] E. Van Gheem, R. Pintelon, A. Hubin, J. Schoukens, P. Verboven, O. Blajiev, J. Vereecken, Electrochim Acta 51 (2006) 1443.

[37] O.L. Blajiev, T. Breugelmans, R. Pintelon, H. Terryn, A. Hubin, Electrochim. Acta 53 (2008) 7451.

[38] Y. Van Ingelgem, E. Tourwé, O.L. Blajiev, R. Pintelon, A. Hubin, Electroanalysis 21 (2009) 730.

[39] M. Itagaki, N. Kobari, S. Yotsuda, K. Watanabe, J. Power Sources 135 (2004) 255.

[40] D.A. Harrington, J. Electroanal. Chem. 355 (1993) 21.

[41] M.E. van der Geest, N.J. Dangerfield, D.A. Harrington, 420 (1997) 89.

[42] M. Labayen, D.A. Harrington, J. Electroanal. Chem., 567 (2004) 185.

[43] F. Seland, R. Tunold, D.A. Harrington, Electrochim. Acta, 51 (2006) 3827.

[44] F. Seland, R. Tunold, D.A. Harrington, Electrochim. Acta, 53 (2008) 6851.

[45] K-K Shiu, Y. Zhang, K-Y Wong, J. Electroanal. Chem. 389 (1995) 105.

[46] Z. Stoynov, Electrochim. Acta 38 (1993) 1919.

[47] C.A. Anastassiou, K.H. Parker, D. O'Hare, Anal. Chem. 77 (2005) 3357.

[48] R.L. Sacci and D.A. Harrington, ECS Trans. 19 (2009) 123.

[49] D.J. Gavaghan, A.M. Bond, J. Electroanal. Chem. 480 (2000) 133.

[50] M.J. Honeychurch, A.M. Bond, J. Electroanal. Chem. 529 (2002) 3.

[51] W.G. Bessler, Solid State Ionics 176 (2005) 997.

[52] L.F. Shampine, R.M. Corless, J. Comp. Appl. Math. 125 (2000) 31.

[53] D.A. Harrington, ECS Trans. 45 (2013) 3.

[54] D.A. Harrington, B.E. Conway, Electrochim. Acta, 32 (1987) 1703.

[55] W.L. Underkofler, I. Shain, Anal. Chem. 37 (1965) 218.

[56] D. Henderson, J.G. Gordon II, J. Electroanal. Chem. 108 (1980) 129.

[57] S.O. Engblom, J.C. Myland and K.B. Oldham, J. Electroanal. Chem. 480 (2000) 120.

[58] J.C. Myland, K.B. Oldham, J. Electroanal. Chem. 535 (2002) 27.

[59] G.C. Temes, J.W. LaPatra, Introduction to Circuit Synthesis and Design, McGraw-Hill, NY, 1977, p. 36. 
[60] J.R. Macdonald, M.K. Brachman, Rev. Mod. Phys. 28 (1956) 393.

[61] C. Gabrielli, M. Keddam, H. Takenouti, in: Electrochemical Impedance: Analysis and Interpretation, ASTM STP 1188, J.R. Scully, D.C. Silverman, M.W. Kendig, eds, ASTM, Philadelphia, 1993, p 140.

[62] A. Sadkowski, Solid State Ionics 176 (2005) 1987.

[63] A. Sadkowski, J.-P. Diard, C. Montella, J. Electrochem. Soc., 156 (2009) F7.

[64] B.A. Boukamp, J. Electrochem. Soc. 142 (1995) 1885.

[65] B.A. Boukamp, Solid State Ionics 169 (2004) 65.

[66] R.L. Sacci, D.A. Harrington, ECS Trans. 19 (2009) 123.

[67] C. Désilets, A. Lasia, Electrochim. Acta 78 (2012) 286.

[68] D. Schuhmann, Electrochim. Acta 35 (1990) 1527.

[69] D.A. Harrington, P. van den Driessche, Electrochim. Acta 56 (2011) 8005.

[70] H. Angerstein-Kozlowska, B.E. Conway, W.B.A Sharp, J. Electroanal. Chem. 43 (1973) 9. 